\title{
Improve Results for Set Identities
}

\author{
Yonghong Liu \\ School of Automation, Wuhan University of Technology, Wuhan, China \\ Email: hylinin@163.com
}

Received 21 December 2013; revised 21 January 2014; accepted 28 January 2014

Copyright (C) 2014 by author and Scientific Research Publishing Inc.

This work is licensed under the Creative Commons Attribution International License (CC BY).

http://creativecommons.org/licenses/by/4.0/

(c) (i) Open Access

\section{Abstract \\ In this work, we presented a new law which was based on the well-known duality property for the set identities. We introduced the diagrams that could be applied to the proof for the set identities. Some prime examples were also provided to illustrate the proposed law.}

\section{Keywords}

\section{Set Theory; Duality Property; Identity}

\section{Introduction}

The set identities are used in many fields, including probability, statistics, propositional logic, Boolean algebra (the binary operations $\vee, \wedge$ and a unary ${ }^{-}$), lattices (the binary operations $\vee$ and $\wedge$ ), BCL/BCL+-algebra [1]-[4] (the binary operation * ) and computer science. De Morgan's laws are a pair of transformation rules that are both valid rules of inference, and Venn diagrams are used to analyze logical arguments and to illustrate relationships between sets.

In the set theory and Boolean algebra, "Identities come in pairs" is now often stated as the relationship between the two identities in each pair we use the concept of a dual, it is certainly nothing new, such as the famous De Morgan laws, dating back to the 19th century, and expresses the duality of the set identities, but it has long been thought that the law of the double complement does not have duality. This is an interesting problem, shedding new light on the operations of sets.

Venn diagrams do not prove whether equality is true or not. So, this is the problem of basic theory, and we are to dope out a solution to the problem.

In this work we present a new law, and we also put the definition of difference evolved into law (i.e. the dual of difference-set law), domination laws and the identity laws. The results show that the diagram can meet proof of sets that this research could be useful to understand the set essence. 


\section{Set Identities}

Table 1 presents some set identities that arise firstly. In general, we have the following law, and we prove that first.

Law 2.1 (Liu’s law). $A \cup(\overline{A \cup B})=A \cup \bar{B}$.

Note that the so called "Liu's law” for propositions, named after the Yonghong Liu.

Proof. We have

$$
\begin{aligned}
A \cup(\overline{A \cup B}) & =A \cup(\bar{A} \cap \bar{B}) \\
& =(A \cup \bar{A}) \cap(A \cup \bar{B}) \\
& =U \cap(A \cup \bar{B}) \\
& =(U \cap A) \cup(U \cap \bar{B}) \\
& =A \cup \bar{B} .
\end{aligned}
$$

The proof is completed.

Corollary 2.1 $A \cap(\overline{A \cap B})=A \cap \bar{B}$ (Difference-set law) and $A \cup(\overline{A \cup B})=A \cup \bar{B}$ (Liu's law) is a dual law (see Table 1).

The Liu's law is to fill gaps in an existing textbook [5]. The most important set identities, such as domination laws and De Morgan’s laws (first De Morgan’s law and second De Morgan’s law) are dual laws.

\section{Diagrams and Definitions}

As we know, Venn diagrams are often used to indicate the relationships among sets. But, it lacks the proof for set identities. New, here's the problem that we confront, and we must change and find new solutions.

Set can be represented graphically using curve. We draw a curved surface to indicate the universal set $U$, which is the set of the curve. We use notation $S$ to denote the curve diagram of the sets, and $S$ is a finite set. In curved surface, let $\cap$ and $U$ be a coordinate set of operation. Let $\wp=(U, S, U, \cap)$ be a operation, then we have the following definitions.

Definition 3.1 Let $A$ and $B$ be sets. Set $B$ is a subset of set $A$, and is denoted by $B \subseteq A$. This is illustrated in Figure 1. That is, if and only if $A \cup B=A$, then $B \subseteq A$.

Definition 3.2 The intersection of the sets $A$ and $B$, denoted by $A \cap B$. This is illustrated in Figure 2.

Definition 3.3 The union of the sets $A$ and $B$, denoted by $A \cup B$. This is illustrated in Figure 3.

Definition 3.4 The Liu's law is illustrated in Figure 4.

Table 1. Set identities.

\begin{tabular}{cc} 
Identity & Name \\
$A \cup(\overline{A \cap B})=U$ & Domination Laws \\
$A \cap(\overline{A \cup B})=\Phi$ & \\
$A \cup(A \cap(\overline{A \cup B}))=A$ & Identity Laws \\
$A \cap(A \cup(\overline{A \cap B}))=A$ & \\
$\overline{(\bar{A})} \cup(A \cap(\overline{A \cup B}))=\overline{(\bar{A})}$ & Complementation Laws \\
$\overline{(\bar{A})} \cap(A \cup(\overline{A \cap B}))=\overline{(\bar{A})}$ & Difference-Set Law \\
$A \cap(\overline{A \cap B})=A \cap \bar{B}$ & Liu’s Law \\
$A \cup(\overline{A \cup B})=A \cup \bar{B}$ & \\
\hline
\end{tabular}




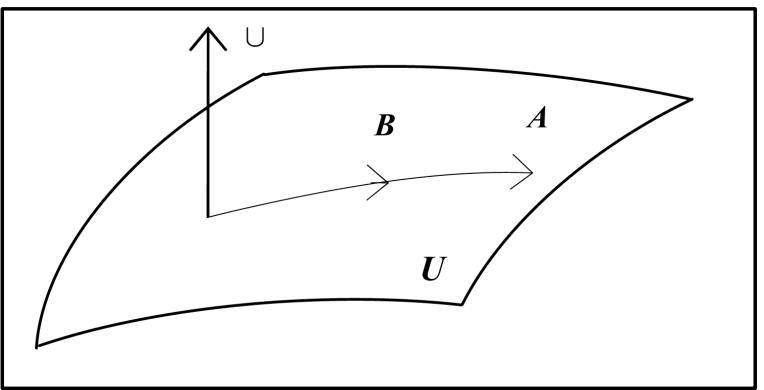

Figure 1. $B \subseteq A$.

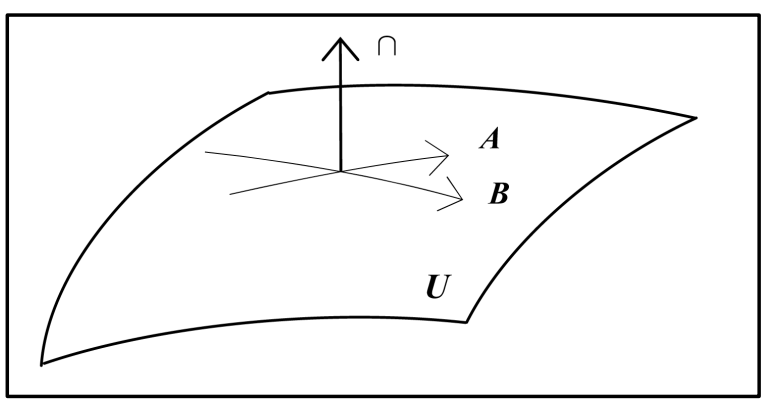

Figure 2. $A \cap B$.

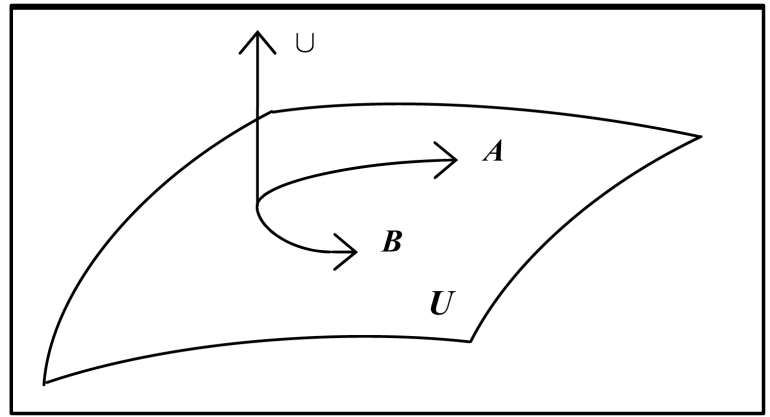

Figure 3. $A \cup B$.

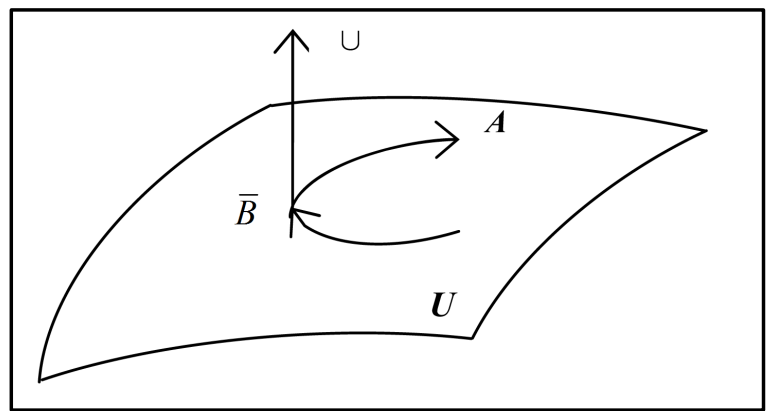

Figure 4. $A \cup \bar{B}$.

Definition 3.5 The difference of the sets $A$ and $B$, denoted by $A-B$. This is illustrated in Figures 5(a) and (b).

Definition 3.6 The complement of the set $A$, denoted by $A$. This is illustrated in Figures 6(a) and (b).

Definition 3.7 The domination laws are illustrated in Figure 7.

Definition 3.8 The domination laws can be decomposed into null set $\Phi$ and universal set $U$. This is illustrated in Figures 8(a) and (b). 


\section{Applications}

Law 4.1 (Associative law). Use diagram to prove that

$$
A \cup(B \cup C)=(A \cup B) \cup C \text {. }
$$

Proof. Let $\wp=(U, S, \cup, \cap)$ be a machine on the set. Let $A, B$, and $C$ be sets with $A, B, C \subseteq S$, and let $\bigcup_{1}, \bigcup_{2}, \bigcup_{3}, \cup_{4} \subseteq \bigcup$. We use the coordinate sets to show that

$$
A \bigcup_{2}\left(B \bigcup_{1} C\right)=\left(A \bigcup_{3} B\right) \bigcup_{4} C .
$$

The associative law is proved in Figures 9(a) and (b). We have (see Figures 9(a) and (b))

Figure 9 displays the graphs of the set $\wp$. Here, if and only if,

$$
\left(\cup_{1}\right) \cup\left(\cup_{2}\right)=\left(\cup_{3}\right) \cup\left(\cup_{4}\right)=\bigcup \text { and }\left(\bigcup_{1}\right) \cap\left(\cup_{2}\right)=\left(\cup_{3}\right) \cap\left(\cup_{4}\right)=U \text {. }
$$

Then $\bigcup_{1}=\bigcup_{2}=\bigcup, \bigcup_{3}=\bigcup_{4}=\bigcup$. Since the curve diagrams for $A \bigcup_{2}\left(B \bigcup_{1} C\right)$ and $\left(A \bigcup_{3} B\right) \bigcup_{4} C$ is same. That is, the two curved surfaces are set-preserving equivalent. The identity of associative law is valid.
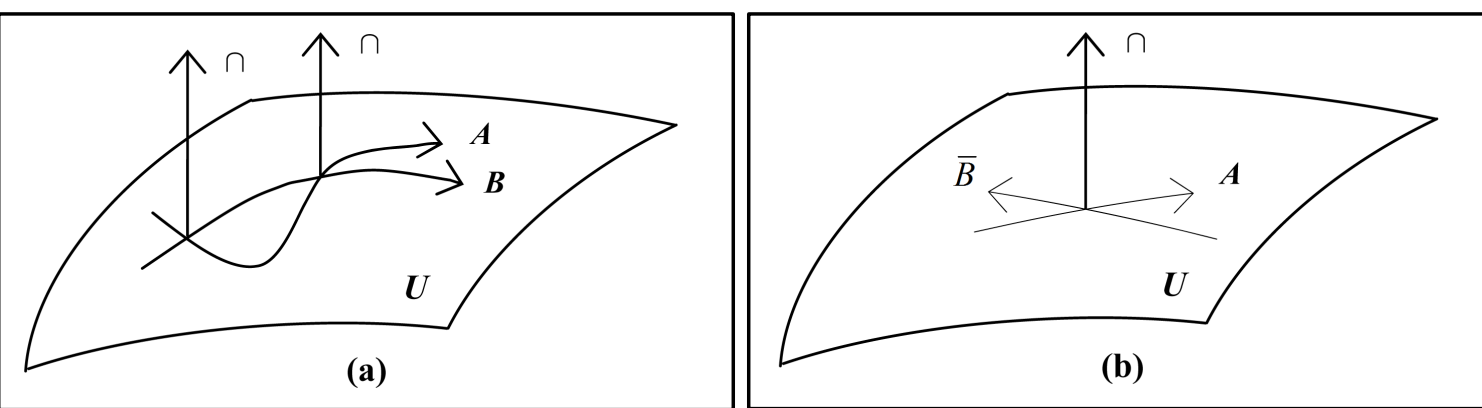

Figure 5. $A-B$. (a) $A \cap(\overline{A \cap B})$; (b) $A \cap \bar{B}$.
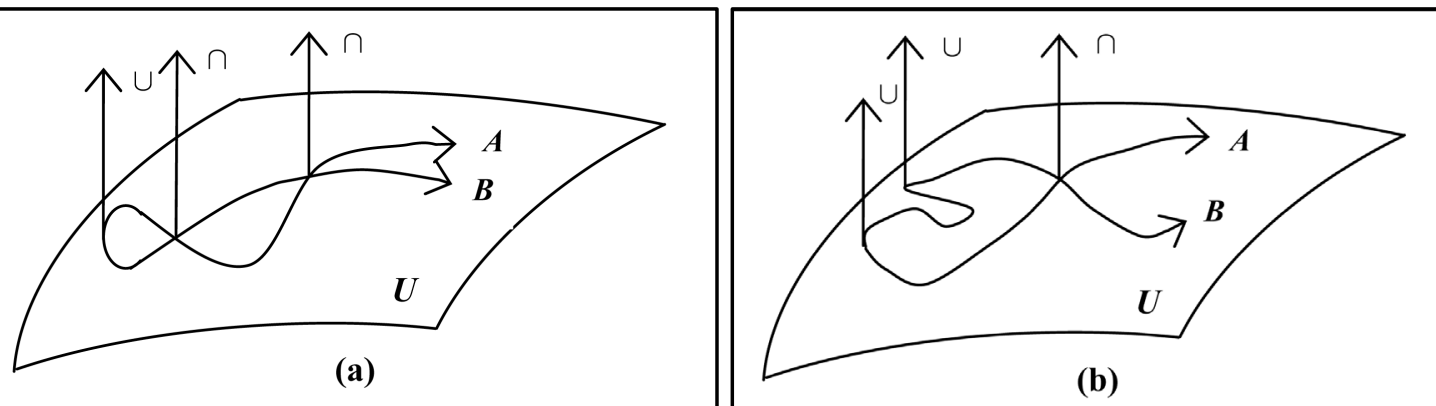

Figure 6. $\bar{A}$. (a) The $U / \cap \cap$ model for $\bar{A}$; (b) The $U U / \cap$ model for $\bar{A}$.

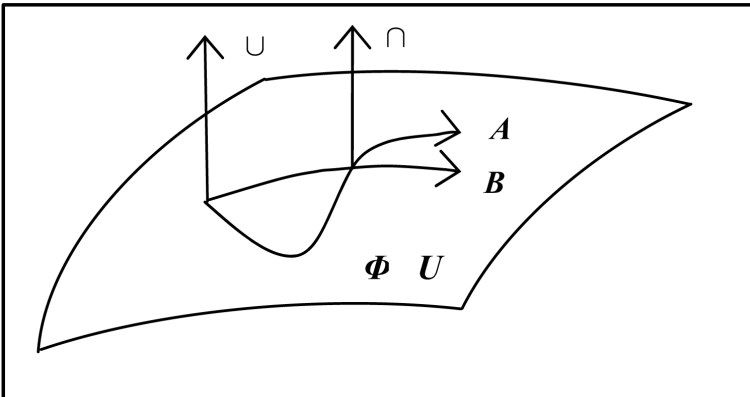

Figure 7. Set of the domination laws. 
Law 4.2 (Distributive law). Use diagram to prove that

$$
A \cap(B \cup C)=(A \cap B) \cup(A \cap C) .
$$

Proof. Let $\wp=(U, S, \cup, \cap)$ be a machine on the set. Let $A, B$, and $C$ be sets with $A, B, C \subseteq S$. The distributive law is proved in Figures 10(a) and (b). We have (see Figures 10(a) and (b))

Where $\bigcap^{\prime}$ and $\bigcap^{\prime \prime}$ be coordinate set of operation with $\bigcap^{\prime}, \bigcap^{\prime \prime} \subseteq \bigcap$, we have

$$
\left(A \cap^{\prime \prime} B\right) \cup\left(A \cap^{\prime} C\right)=(A \cap B) \cup(A \cap C) .
$$

If and only if,

$$
\left(\cap^{\prime}\right) \cup\left(\cap^{\prime \prime}\right)=\cap \text { and }\left(\cap^{\prime}\right) \cap\left(\cap^{\prime \prime}\right)=\cap,
$$

then $\bigcap^{\prime}=\bigcap^{\prime \prime}=\bigcap$. Hence, by Figure 10(a), we have

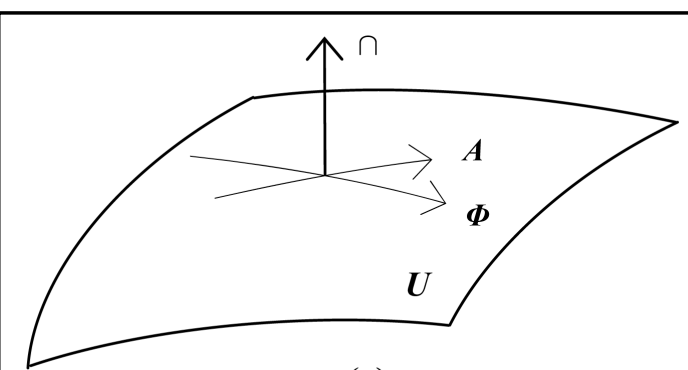

(a)

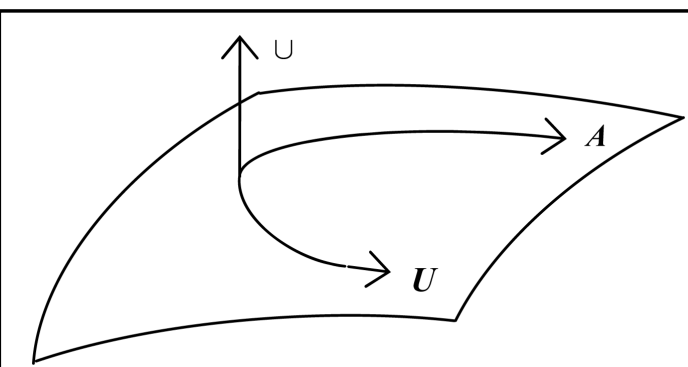

(b)

Figure 8. $\Phi$ and $U$. (a) The ith term of $\Phi$; (b) The ith term of $U$.

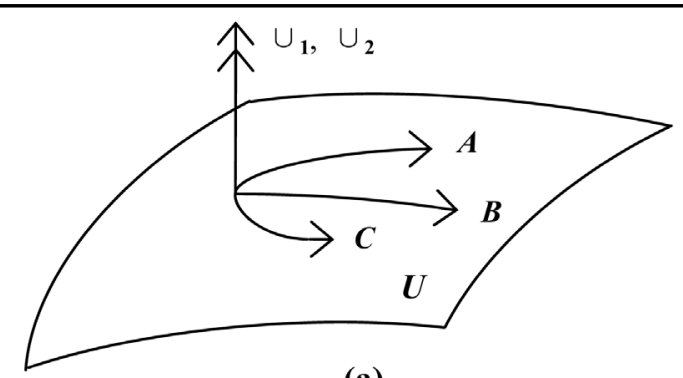

(a)

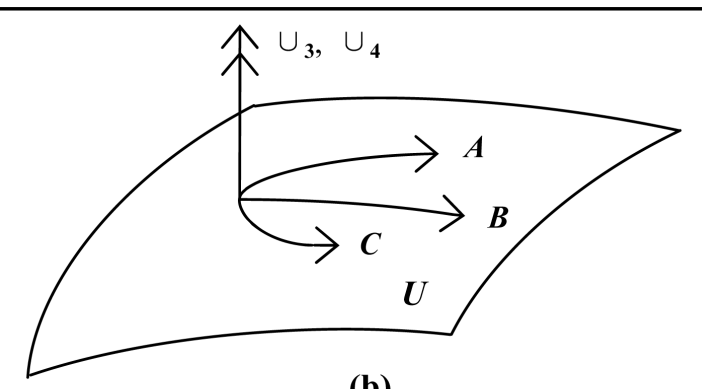

(b)

Figure 9. Set of the associative law. (a) $A \bigcup_{2}\left(B \bigcup_{1} C\right)$; (b) $\left(A \bigcup_{3} B\right) \bigcup_{4} C$.

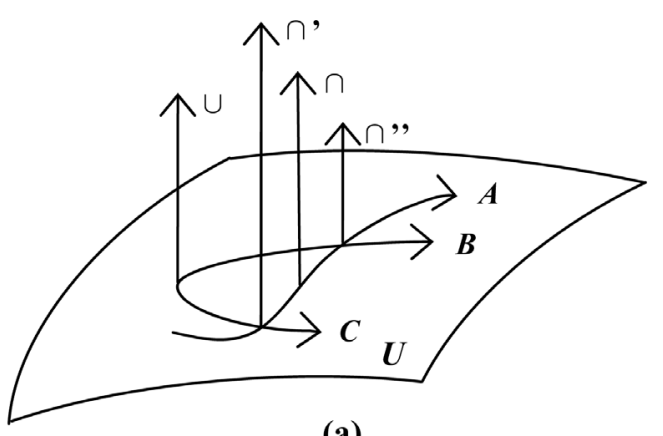

(a)

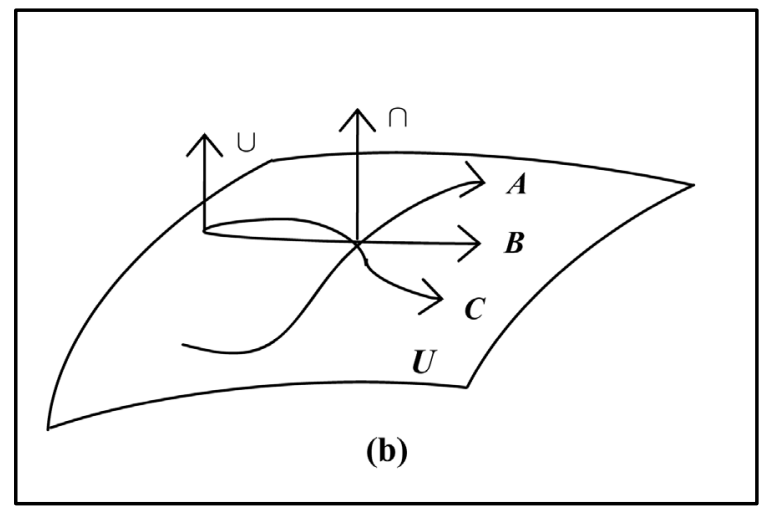

Figure 10. Set of the distributive law. (a) $A \cap(B \cup C)$; (b) $(A \cap B) \cup(A \cup C)$. 


$$
A \cap(B \cup C)=\left(A \cap^{\prime \prime} B\right) \cup\left(A \cap^{\prime} C\right)
$$

This is what we needed to prove.

Example 4.1 Use set identities to prove that

$$
\left\{\begin{array}{l}
x \cap(\overline{A \cap B})=\bar{B} \\
A \cap(\bar{B} \cup x)=x
\end{array}\right.
$$

Whether the system has a unique solution

$$
x=A \cap \bar{B} .
$$

Proof. Let $x \cap(\overline{A \cap B})=\bar{B}$ be equivalent to saying that two equations

thus

Let $A \cap(\bar{B} \cup x)=x$ we also have

$$
\begin{aligned}
x \cap(\overline{A \cap B}) & =x \cap(\bar{A} \cup \bar{B}) \\
& =(x \cap \bar{A}) \cup(x \cap \bar{B}) \\
& =\bar{B} \cup \bar{B} \\
& =\bar{B} .
\end{aligned}
$$

thus

$$
\left\{\begin{array}{l}
A \cap \bar{B}=x \\
A \cap x=x .
\end{array}\right.
$$

$$
\begin{aligned}
A \cap(\bar{B} \cup x) & =A \cap(\bar{B} \cup(A \cap \bar{B})) \\
& =A \cap \bar{B} \\
& =x,
\end{aligned}
$$

and thus

$$
\begin{aligned}
A \cap(A \cap \bar{B}) & =(A \cap A) \cap \bar{B} \\
& =A \cap \bar{B} \\
& =x .
\end{aligned}
$$

Conversely, if $x=A \cap \bar{B}$, then

$$
\begin{aligned}
x \cap(\overline{A \cap B}) & =(A \cap \bar{B}) \cap(\overline{A \cap B}) \\
& =((\overline{A \cap B}) \cap A) \cup((\overline{A \cap B}) \cap \bar{B}) \\
& =(A \cap \bar{B}) \cup((\bar{A} \cup \bar{B}) \cap \bar{B}) \\
& =(A \cap \bar{B}) \cup \bar{B} \\
& =\bar{B} .
\end{aligned}
$$

thus

$$
\begin{aligned}
A \cap(\bar{B} \cup x) & =A \cap(\bar{B} \cup(A \cap \bar{B})) \\
& =A \cap \bar{B} \\
& =x .
\end{aligned}
$$

Example 4.1 is proved. 
Example 4.2 Use set identities to prove that

$$
\left\{\begin{array}{l}
x \cup(A \cap B)=\overline{A+B} \\
x \cup A=A \cup \bar{B} .
\end{array}\right.
$$

Whether the system has a unique solution

$$
x=\overline{A \cup B} .
$$

Proof. Let $x \cup(A \cap B)=\overline{A+B}$ be equivalent to saying that two equations

$$
\left\{\begin{array}{l}
x=(\overline{A+B}) \cap V \\
A \cap B=(\overline{A+B} \cap \bar{V}) .
\end{array}\right.
$$

where for all $V$ in $U$ we have

$$
\begin{aligned}
x \cup(A \cap B) & =((\overline{A+B}) \cap V) \cup((\overline{A+B}) \cap \bar{V}) \\
& =(\overline{A+B}) \cap(V \cup \bar{V}) \\
& =(\overline{A+B}) \cap U \\
& =\overline{A+B} .
\end{aligned}
$$

Let $\quad x \cup A=A \cup \bar{B}$ we also have

$$
\left\{\begin{array}{l}
x=(A \cup \bar{B}) \cap W \\
A=(A \cup \bar{B}) \cap \bar{W} .
\end{array}\right.
$$

where for all $W$ in $U$ we have

$$
\begin{aligned}
x \cup A & =((A \cup \bar{B}) \cap W) \cup((A \cup \bar{B}) \cap \bar{W}) \\
& =(A \cup \bar{B}) \cap(W \cup \bar{W}) \\
& =(A \cup \bar{B}) \cap U \\
& =A \cup \bar{B} .
\end{aligned}
$$

Conversely, if $x=\overline{A \cup B}$, then

$$
\begin{aligned}
x \cup(A \cap B) & =(\overline{A \cup B}) \cup(A \cap B) \\
& =\overline{(A \cup B) \cap(\overline{A \cap B})} \\
& =\overline{(A \cup B) \cap(\bar{A} \cup \bar{B})} \\
& =\overline{A+B} .
\end{aligned}
$$

and thus

$$
x \bigcup A=(\overline{A \cup B}) \cup A=A \bigcup \bar{B}
$$

The proof is complete.

\section{References}

[1] Liu, Y.H. (2011) A New Branch of the Pure Algebra: BCL-Algebras. Advances in Pure Mathematics, 1, $297-299$. http://dx.doi.org/10.4236/apm.2011.15054

[2] Liu, Y.H. (2012) On BCL ${ }^{+}$-Algebras. Advances in Pure Mathematics, 2, 59-61. 
http://dx.doi.org/10.4236/apm.2012.21012

[3] Deena, A.K. and Rodyna, H. (2013) On BCL-Algebra. Journal of Advances in Mathematics, 3, 184-190.

[4] Liu, Y.H. (2013) Partial Orders in $\mathrm{BCL}^{+}$-Algebra. Journal of Advances in Mathematics, 5, 630-634.

[5] Rosen, K.H. (1998) Discrete Mathematics and Its Applications. 4th Edition, McGraw-Hill, New York. 